\title{
Pairing competition between metacentric and telocentric chromosomes in autotetraploid rye
}

\author{
Elena Benavente and \\ Juan Orellana*
}

Departamento de Genética, Facultad de Biología, Universidad Complutense, 28040 Madrid, Spain.

* Departamento de Genética, E.T.S.I. Agrónomos, Universidad Politécnica, 28040 Madrid, Spain.

Pairing preferences have been analyzed in tetraploid cells of rye telocentric substitution lines where competition took place between telocentric and metacentric partners within each set. Chromosome arms in telocentric condition (bearing large telomeric blocks of C-heterochromatin) showed a higher probability of being associated at metaphase I.

However, the excess of identical over homologous pairing found in most cases could not be ascribed to such differences in pairing (or chiasma formation) ability between homologues. Other hypotheses for explaining preferential pairing in polyploids are discussed.

\section{INTRODUCTION}

When more than two homologous chromosomes are present in the same nucleus, a choice has to be made between potential pairing partners. Many studies have attempted to analyze pairing preferences in polyploids where competition introduces an entirely new dimension in the meiotic process. In most of these studies specific chromosomes could not be distinguished since conventional staining methods were used; then the development of theoretical models which use pooled data of all chromosomes was needed to investigate meiotic behaviour in competitive situations. These models predict the frequencies of the different meiotic configurations (multivalents, bivalents and univalents) assuming random pairing between all partners (John and Henderson, 1962; Sved, 1966; Sybenga, 1975; Jackson and Hauber, 1982). Deviations from the expected ratios have been taken as indirect evidence of preferential pairing.

Proposed theoretical models are often severely restrictive because (i) they usually assume that all chromosomes of different sets and all chromosomes within a given set have the same probability of pairing and (ii) only one synaptic initiation site located at the end of each chromosome arm is considered. Although these premises cannot be easily verified those models are the only available method of analysis when conventional staining techniques are employed.

Recently, the use of C-bands as cytological markers has allowed direct analyses of pairing preferences for specific chromosome arms in tetraploid meiocytes of rye and grasshoppers (Giraldez and Santos 1981; Santos et al., 1983; Naranjo and Orellana, 1984; Orellana and Santos, 1985; Benavente and Orellana, 1986). Orellana and Santos (1985) pointed out that mutual influences between both arms should be taken into account when metacentric or submetacentric chromosomes are considered because pairing preferences at a given arm might influence those of the other arm. Moreover, when pairing preferences were analyzed in telotrisomics of rye where three homologous $1 \mathrm{RS}$ chromosome arms were in competition, an excess of metaphase I associations involving the short arms of the two metacentric partners was found in all plants (Benavente and Orellana, 1985). It was attributed to the high probability for pairing of the long arm (in disomic condition) which could facilitate the progress of synapsis along the chromosome and, consequently, the frequency of pairing between the short arms of metacentric partners would be increased. So, inter-arm effects may lead even to artificial preferential pairing for specific chromosome arms.

In this paper we analyze pairing competition in tetraploid cells of plants where one homologue of 
certain pairs was substituted by their corresponding two telos. The chromosomal constitution of these plants should allow the detection of inter-arm effects (if they exist), their partitioning from other effects and estimation of their magnitudes.

\section{MATERIALS AND METHODS}

A single plant of line $t$ (with partly "Petkus Spring" rye background and kindly supplied by Dr Sybenga, Agricultural University of Wageningen, The Netherlands) was employed in the present study as the source of telocentric chromosomes. This plant, which had one $1 R$ and both $3 R$ homologues substituted by their ${ }^{4}$ corresponding two telos (1R heterozygous and $3 \mathrm{R}$ homozygous telocentric substitutions, according to Sybenga (1975)), was crossed with one plant of the inbred line $\mathrm{E}$ of diploid rye. As described in a previous work (see Benavente and Orellana, 1985), the C.banding pattern of line $t$ is characterized by the presence of conspicuous blocks of constitutive heterochromatin at the telomeres in both normal (metacentric) and telocentric $1 \mathrm{R}$ and $3 \mathrm{R}$ chromosomes, whereas such C-bands are absent in these chromosomes from the inbred line $\mathrm{E}$ (see fig. 1). $\mathrm{F} 1$ plants heterozygous for the $1 \mathrm{R}$ and $3 \mathrm{R}$ telocentric substitutions were selected to perform this study.

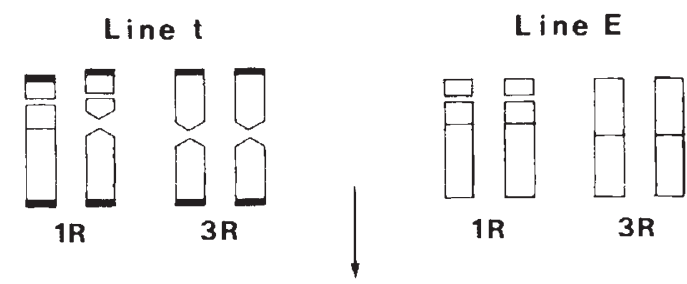

tE $2 x$

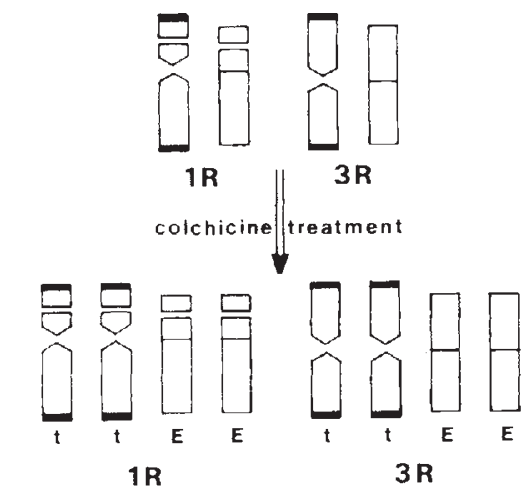

Figure 1 Chromosome constitution and C-banding pattern for chromosomes $\mathrm{R}$ and $3 \mathrm{R}$ in tetraploid cells of the telocentric substitution heterozygotes of rye $t E$.
For mitotic analysis, seeds were germinated on wet filter paper in Petri dishes at room temperature. When primary roots were $1-2 \mathrm{~cm}$ long their tips were excised and immersed in tap water at $0^{\circ} \mathrm{C}$ to shorten the chromosomes. Subsequently, they were fixed in $1: 3$ acetic ethanol.

At the three-leaf stage, seedlings were treated with colchicine using a technique based on that described by Thomas and Pickering (1979) as follows. Plantlets were dug up and roots were immersed in a 0.15 per cent colchicine plus 0.2 per cent DMSO (dimethylsulphoxide) solution for 1 hour; then roots were cut away at about $2 \mathrm{~cm}$ and reimmersed for 5 hours. After washing in tap water they were replanted. Some of the treated plantlets turned out to be diploid/tetraploid chimeras. Anthers of the emerging spikes were stained in 2 per cent acetic orcein to determine their diploid or tetraploid chromosome constitution. Tetraploid anthers were fixed in $1: 3$ acetic ethanol and stored at $4^{\circ} \mathrm{C}$ for several months.

The fixed material (root tips and anthers) was squashed following the Giemsa C-banding technique described previously (Giraldez et al., 1979).

\section{RESULTS}

The special staining characteristics of the nucleolus organizer region located in the $1 \mathrm{RS}$ chromosome arm and differences in their relative lengths make possible an undoubted identification of both arms of chromosome $1 \mathrm{R}$. For chromosome $3 \mathrm{R}$ the situation is not so easy since other members of the complement have similar lengths and centromere positions, in addition to the fact that its short and long arms show only slight differences in their relative lengths. For these reasons, the use of a differential staining technique like C-banding has been necessary to recognize both $3 \mathrm{R}$ chromosome arms, either in the metacentric or telocentric condition.

Pairing preferences for specific arms can be analyzed in autotetraploids by the employment of any marker which differentiates cytologically among the four doses of each chromosome arm. All diploid plants used here were heterozygotes both for telomeric C-bands and telocentric chromosomes (see fig. 1). In tetraploid cells obtained after colchicine treatment each chromosome is accompanied by one identical and two homologous but not identical partners. Sets 1R and $3 \mathrm{R}$ contain two identical normal (metacentric) chromosomes, without telomeric heterochromatin, and two identical ones substituted by their corre- 

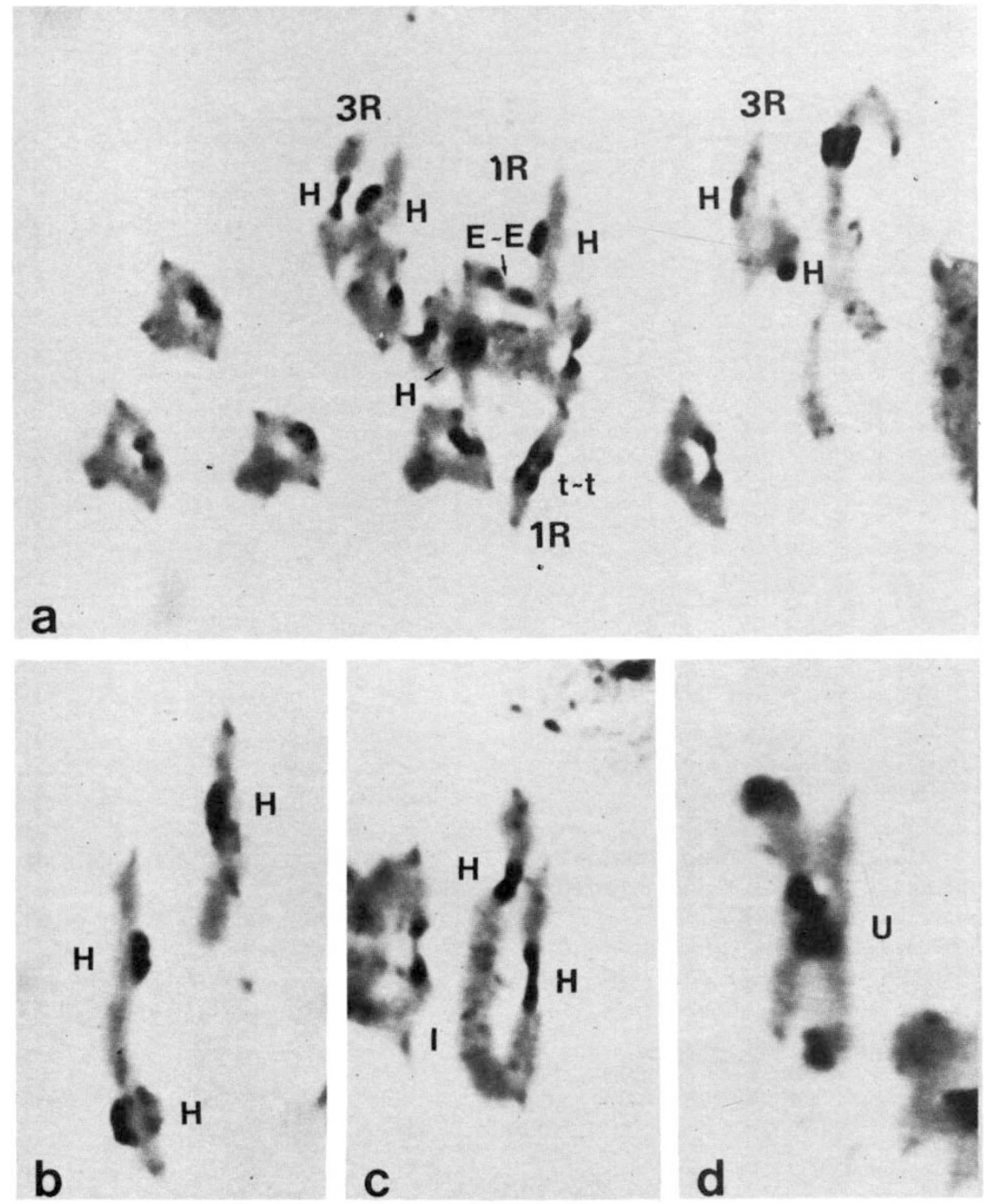

Figure 2 Meiotic configurations for chromosomes $1 R$ and $3 R$ in tetraploid cells of plants $t E$. (a) C-banded metaphase I tetraploid cell with one $1 R$ chain quadrivalent, one $1 R S$ telocentric bivalent and two $3 R$ V-shaped trivalents. The type of pairing for the marked arms is as follows: identical E-E and $t-t$ for IRS (2I, as noted in table 1), and homologous $H$ for $1 R L$ (2H), 3RS (2H) and $3 \mathrm{RL}(2 \mathrm{H})$. (b) $1 \mathrm{R} \mathrm{V}$-shaped trivalent with homologous pairing for the short and long arms and $1 \mathrm{R}$ heteromorphic bivalent with homologous pairing for the short arm. (c) $3 R$ chain quadrivalent with homologous pairing for the short arm and identical pairing for the long arm. (d) $1 \mathrm{R}$ X-shaped quadrivalent with undetermined pairing (U4) for the long arm.

sponding telos, with prominent C-bands at the telomeres, derived from lines $\mathrm{E}$ and $\mathrm{t}$, respectively (fig. 1).

The use of such cytological markers has allowed identification of the type of pairing (between identical or homologous non-identical partners) for the marked arms in all metaphase I configurations, except in those in which three or four chromosomes were associated at the same arm (undetermined pairing (fig. 2)). Table 1 shows the frequencies of the different kinds of tetraploid metaphase I cells observed for both arms of chromosomes $1 \mathrm{R}$ and $3 \mathrm{R}$. The bound arm frequencies, estimated as the minimum number of chias- 
Table 1 Distribution of metaphase I cells according to their type of pairing for the marked arms. The mean number of bound arms per cell $(\mathrm{ba} / \mathrm{cel}$.) is also included

Chromosome 1R

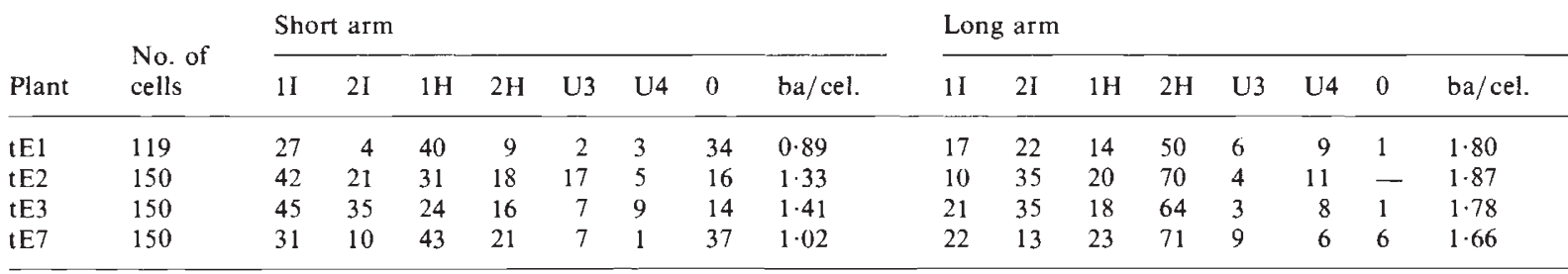

Chromosome 3R

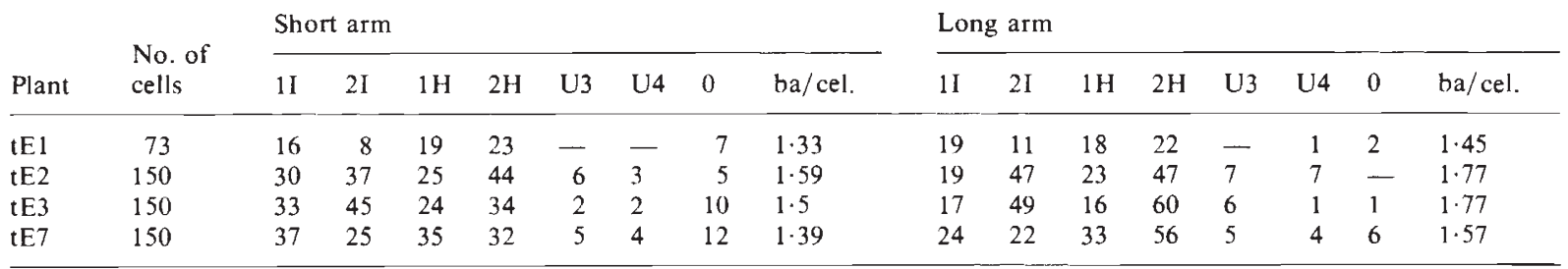

I: cells with one (1I) or two (2I) bonds between identical partners; H: cells with one (1H) or two (2H) bonds between homologous partners; U: cells where three (U3) or four (U4) chromosomes were associated at the marked arm; 0: cells with the four partners unpaired.

mata necessary to explain each meiotic configuration, has also been included.

Two types of pairing involving either metacentric $(E-E)$ or telocentric $(t-t)$ chromosome arms can be distinguished in cells with identically paired partners (for an example, see $1 \mathrm{RS}$ in fig. $2 \mathrm{a}$ ). The ratio $\mathrm{E}-\mathrm{E}: \mathrm{t}-\mathrm{t}$ is expected to be $1: 1$ assuming the same probability of both types of identical pairing. An excess of $t-t$ over $E-E$ identical associations has been found in all cases, with significant deviations from the ratio 1:1 in five comparisons (table 2).

In addition, there are four possible pairwise combinations between homologous partners involving one metacentric and one telocentric chromosome arm (t-E). Therefore a ratio of $1: 1: 4$ of $E-E: t-t: t-E$ is expected with random pairing among the four chromosomes of each set when

Table 2 Numbers of the two different types of identical pairing involving either metacentric (E-E) or telocentric ( $t-t)$ chromosome arms. Expected values have been calculated under a ratio $1: 1 \mathrm{E}-\mathrm{E}: \mathrm{t}-\mathrm{t}$

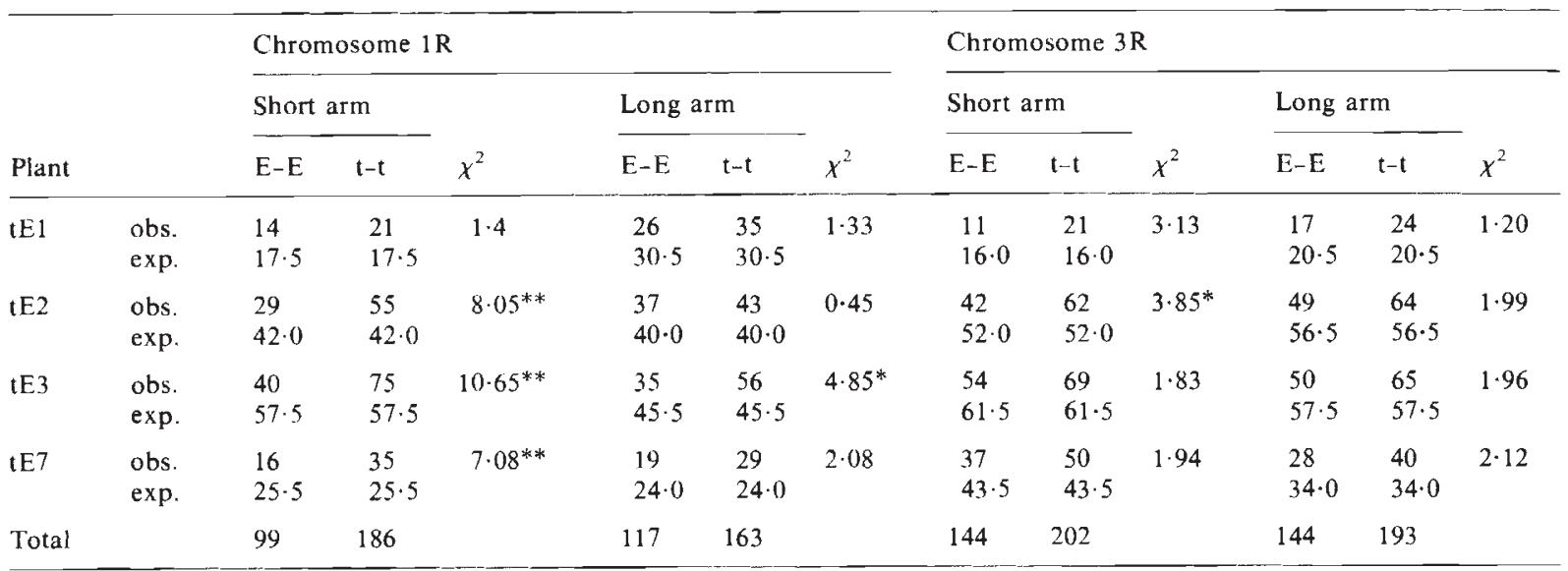

* Significant at the level of 5 per cent.

** Significant at the level of 1 per cent. 
Table 3 Numbers of cells with at least one identical or homologous bond at metaphase I for both arms of chromosomes $1 \mathrm{R}$ and 3R. Expected values have been calculated under a ratio $1: 2 \mathrm{I}: \mathrm{H}$

\begin{tabular}{|c|c|c|c|c|c|c|c|c|c|c|c|c|c|}
\hline \multirow[b]{3}{*}{ Plant } & & \multicolumn{6}{|c|}{ Chromosome 1R } & \multicolumn{6}{|c|}{ Chromosome $3 \mathrm{R}$} \\
\hline & & \multicolumn{2}{|c|}{ Short arm } & \multirow[b]{2}{*}{$x^{2}$} & \multicolumn{2}{|c|}{ Long arm } & \multirow[b]{2}{*}{$x^{2}$} & \multicolumn{2}{|c|}{ Short arm } & \multirow[b]{2}{*}{$x^{2}$} & \multicolumn{2}{|c|}{ Long arm } & \multirow[b]{2}{*}{$x^{2}$} \\
\hline & & $I$ & $H$ & & $I$ & $H$ & & $I$ & $H$ & & $I$ & $H$ & \\
\hline $\mathrm{tE} 1$ & $\begin{array}{l}\text { obs. } \\
\text { exp. }\end{array}$ & $\begin{array}{l}31 \\
26 \cdot 67\end{array}$ & $\begin{array}{l}49 \\
53 \cdot 33\end{array}$ & $1 \cdot 06$ & $\begin{array}{l}39 \\
34 \cdot 33\end{array}$ & $\begin{array}{l}64 \\
68 \cdot 67\end{array}$ & 0.95 & $\begin{array}{l}24 \\
22 \cdot 0\end{array}$ & $\begin{array}{l}42 \\
44 \cdot 0\end{array}$ & $0 \cdot 27$ & $\begin{array}{l}30 \\
23 \cdot 33\end{array}$ & $\begin{array}{l}40 \\
46 \cdot 67\end{array}$ & $2 \cdot 86$ \\
\hline $\mathrm{tE} 2$ & $\begin{array}{l}\text { obs. } \\
\text { exp. }\end{array}$ & $\begin{array}{l}63 \\
37 \cdot 33\end{array}$ & $\begin{array}{l}49 \\
74 \cdot 67\end{array}$ & $26 \cdot 47^{* *}$ & $\begin{array}{l}45 \\
45 \cdot 0\end{array}$ & $\begin{array}{l}90 \\
90 \cdot 0\end{array}$ & $0 \cdot 0$ & $\begin{array}{l}67 \\
45 \cdot 33\end{array}$ & $\begin{array}{l}69 \\
90 \cdot 67\end{array}$ & $15 \cdot 53^{* *}$ & $\begin{array}{l}66 \\
45 \cdot 33\end{array}$ & $\begin{array}{l}70 \\
90 \cdot 67\end{array}$ & $14 \cdot 13^{* *}$ \\
\hline tE3 & $\begin{array}{l}\text { obs. } \\
\text { exp. }\end{array}$ & $\begin{array}{l}80 \\
40 \cdot 0\end{array}$ & $\begin{array}{l}40 \\
80 \cdot 0\end{array}$ & $60 \cdot 0^{* *}$ & $\begin{array}{l}56 \\
46 \cdot 0\end{array}$ & $\begin{array}{l}82 \\
92 \cdot 0\end{array}$ & $3 \cdot 26$ & $\begin{array}{l}78 \\
45 \cdot 33\end{array}$ & $\begin{array}{l}58 \\
90 \cdot 67\end{array}$ & $35 \cdot 31^{* *}$ & $\begin{array}{l}66 \\
47 \cdot 33\end{array}$ & $\begin{array}{l}76 \\
94.67\end{array}$ & $11 \cdot 04^{* *}$ \\
\hline tE7 & $\begin{array}{l}\text { obs. } \\
\text { exp. }\end{array}$ & $\begin{array}{l}41 \\
35 \cdot 0\end{array}$ & $\begin{array}{l}64 \\
70 \cdot 0\end{array}$ & $1 \cdot 54$ & $\begin{array}{l}35 \\
43 \cdot 0\end{array}$ & $\begin{array}{l}94 \\
86.0\end{array}$ & $2 \cdot 23$ & $\begin{array}{l}62 \\
43 \cdot 0\end{array}$ & $\begin{array}{l}67 \\
86 \cdot 0\end{array}$ & $12 \cdot 59^{* *}$ & $\begin{array}{l}46 \\
45 \cdot 0\end{array}$ & $\begin{array}{l}89 \\
90 \cdot 0\end{array}$ & 0.03 \\
\hline
\end{tabular}

$I=$ cells with identical pairing; $H$ : cells with homologous pairing.

** Significant at the level of 1 per cent.

the three types of associations are considered and consequently, a ratio $1: 2$ identical $(E-E+t-t)$ vs. homologous ( $t-E)$ pairing should be observed.

The total number of bonds between identical or homologous partners or the number of metaphase I cells with identical or homologous pairing might be employed to perform the comparison between observed and expected values. Because the type of pairing is not independent for two bonds within a specific cell (i.e., when one association involves two identical or homologous chromosomes, a second potential bond between the remaining two partners necessarily will show the same type of pairing), the numbers of cells with at least one identical or homologous bond were used here.

An excess of identical pairing from the ratio $1: 2 \mathrm{I}: \mathrm{H}$ has been found in most cases, the differences between observed and expected frequencies being highly significant in particular for the short arms of chromosomes $1 \mathrm{R}$ and $3 \mathrm{R}$ (table 3 ).

Pairing preferences should be studied at pachytene, when synapsis reaches its maximum; but usually only later stages as diakinesis or metaphase I are accessible. The distribution of the different kinds of meiotic configurations and therefore the type of chromosome association at these stages depends upon the patterns of chromosome pairing and the frequency and location of chiasmata. For that reason we cannot discern if the preferences detected here are due to preferences in pairing or preferences in chiasma formation or both. Therefore, the term "preferential pairing" will be considered in the further discussion as preferences for the pairing that is effective for crossing over.

\section{DISCUSSION}

It has been well established in a large number of organisms that diploid chiasma frequency is directly related to bivalent size when different members of the complement are compared, but few attempts have been made to study this point in higher ploidy levels. From the results shown in table 1 there seems to be clear a relationship between the relative lengths of the marked chromosome arms $(1 \mathrm{RS}<3 \mathrm{RS}<3 \mathrm{RL}<1 \mathrm{RL}$; see Schlegel et al., 1987) and their mean chiasma frequency per tetraploid cell. These results are in agreement with those found by John and Henderson (1962) in Schistocerca paranensis and Orellana and Santos (1985) in autotetraploid rye.

For explaining pairing preferences in competitive conditions it has been proposed that hypothetical synaptic initiators (Doyle, 1979) or zygomeres (Sybenga, 1966) exist which would determine the pairing activity of specific chromosomes. Differences in the number or activity of such entities would lead to a different pairing probability for each chromosome and, consequently, to deviations from random for the different types of pairing that can be established between all partners in competition, i.e., associations involving more active partners would be more frequent. The validity of this hypothesis on the origin of nonrandom pairing can only be tested if one is able to determine the actual probability for pairing of specific chromosomes and make a comparison between homologues.

Identical chromosomes are replicas of a single molecule of DNA. Then they cannot differ with respect to any kind of synaptic initiators when 
analyzed in the same generation of colchicine treatment. (Differences between originally identical copies may arise from recombination processes in the offspring of a colchicine-induced autotetraploid.) Therefore it can be assumed that there are no differences in pairing activity (or ability) between identical chromosome arms and the relative frequencies of the two types of identical associations ( $t-t$ and $E-E$ ) can be used as a measure of the synaptic activity of each chromosome ( $t$ and E) since they will reflect faithfully differences of pairing abilities between homologues. Thus the excess of $t-t$ identical associations detected in all plants $\mathrm{tE}$ (table 2) seems to indicate that $\mathrm{t}$ chromosome arms are more active for pairing when compared with $\mathrm{E}$ chromosomes, differences between both homologue types being specially remarkable for short arm 1RS and, in a lower degree, for short arm 3RS.

Besides the hypothetical existence of any kind of zygomeres or synaptic units for explaining differential pairing abilities between $t$ and $E$ chromosomes, other factors may be taken into account to be responsible for the excess of $t-t$ over E-E identical associations.

It has been mentioned above that there exist a relationship between chromosome size and chiasma frequency. In spite of the lack of crossing over and chiasma formation in heterochromatin compared to euchromatin (see Comings, 1972 for a review), there have been several reports of synaptonemal complex in heterochromatin (Stack, 1984); then the pachytene bivalent length may be increased in chromosomes bearing large blocks of C-heterochromatin. Moreover, pairing initiation sites have been reported at the heterochromatic telomeres of certain chromosomes of rye (Abirached-Darmency et al., 1983; Gillies, 1985). Thus the excess of $t-t$ identical associations might be ascribed to the presence of conspicuous telomeric C-bands in chromosomes from line $t$ since effective length for synapsis and therefore pairing ability would be higher than in E chromosomes which lack C-heterochromatin. This effect of C-heterochromatin (length effect) must be dependent on the actual length of each specific chromosome arm, i.e., the increase in pairing ability due to the presence of an heterochromatic block will be relatively greater for short than long arms within the same chromosome, in agreement with the special remarkable deviations from the ratio $1: 1 \mathrm{E}-\mathrm{E}: \mathrm{t}-\mathrm{t}$ obtained for the short arms of chromosomes $1 \mathrm{R}$ and $3 \mathrm{R}$ (table 2). This could be also the explanation for the excess of het-het associations found for chromosome arm 1RS in tetraploid cells of rye where competition took place among metacentric partners (see table 4 in Orellana and Santos, 1985).

It cannot be excluded that higher $t-t$ association frequencies might reflect an effect of structural change on chiasma frequency, as demonstrated for different structurally-rearranged chromosomes in Hypochoeris (Parker et al., 1982; Parker, 1987).

On the other hand, it may be noted here that any inter-arm effect of the progression of pairing as proposed in telotrisomics of rye (Benavente and Orellana, 1985, 1986) should increase the frequency of associations between $E$ chromosome arms, in metacentric condition. If such an effect exists in our material, it is masked by other factors.

Whatever the origin of differential pairing (or chiasma formation) abilities between homologues, they are leading to an excess of associations involving more active partners $(t-t)$ in the autotetraploids analyzed here. Moreover, they could be responsible for the identical over homologous preferential pairing detected in those cases where deviations from the expected ratio $1: 1 \mathrm{E}-\mathrm{E}: \mathrm{t}-\mathrm{t}$ were found, i.e., chromosome arm $1 \mathrm{RS}$ in plants $\mathrm{tE} 2$ and $\mathrm{tE} 3$ and chromosome arm $3 R S$ in plant tE2 (see tables 2 and 3 ). If pairing preferences mainly depend on such differences one could also explain the fit with the expected identical and homologous frequencies assuming random pairing $(I=1 / 2 H)$ when $t$ and $\mathrm{E}$ chromosome arms show similar pairing probabilities $(\mathrm{E}-\mathrm{E}=\mathrm{t}-\mathrm{t})$, i.e., $1 \mathrm{RS}$ in $\mathrm{tE} 1 ; 1 \mathrm{RL}$ in $\mathrm{tE} 1, \mathrm{tE} 2$ and $\mathrm{tE} 7$; $3 \mathrm{RS}$ in tE1; 3RL in tE1 and tE7 (tables 2 and 3 ). However, the remaining results obtained $(\mathrm{E}-\mathrm{E}=\mathrm{t}-\mathrm{t}$ but $I>1 / 2 \mathrm{H}$ and $\mathrm{t}-\mathrm{t}>\mathrm{E}-\mathrm{E}$ but $I=1 / 2 H$ ) cannot be explanable under this assumption. A further analysis should then be made to prove its presumptive validity in the first mentioned case $(\mathrm{t}-\mathrm{t}>\mathrm{E}-\mathrm{E}$ and $I>1 / 2 H)$.

A ratio of $1: 4$ of $\mathrm{E}-\mathrm{E}: \mathrm{t}-\mathrm{E}$ (derived from $I(\mathrm{t}-\mathrm{t}+$ $\mathrm{E}-\mathrm{E}): H(\mathrm{t}-\mathrm{E}) 1: 2$ and $\mathrm{t}-\mathrm{t}: \mathrm{E}-\mathrm{E} 1: 1)$ is expected if the identical preferential pairing detected in those cases arises from a higher pairing (or chiasma formation) probability for $t$ chromosomes only when they are identically associated $(\mathrm{t}-\mathrm{t})($ e.g., this would be the situation if the presence of a telomeric C-band increases the effective pairing length of $t$ chromosomes; obviously, when they pair with an homologous partner ( $t-E)$ the effectivity would be similar to that expected from the association between two chromosome arms which lack Cbands (E-E) since the heterochromatic C-block does not correspond to any homologous region in the euchromatic partner). By contrast, associations involving homologous partners would be more 
frequent if $\mathrm{t}$ chromosome arms show a higher ability for pairing (or chiasma formation) with any other ( $\mathrm{t}$ or $\mathrm{E}$ ). However, table 4 shows that deviations from the ratio $1: 4 \mathrm{E}-\mathrm{E}: \mathrm{t}-\mathrm{E}$ are always due to an excess of pairing between less active partners (E-E). This indicates that differences in pairing activity (or ability) between partners in competition are unable to explain the preferential behaviour detected here, where the association between identical chromosomes seems to be favoured over the homologous pairing even for less active partners.

Table 4 Comparison between the frequencies of associations involving identical less active (E-E) or homologous ( $t-E$ ) partners in those cases wherè the excess of one type of identical pairing ( $t-t>E-E$, table 2 ) was accompanied by an excess of identical over homologous pairing $(I>1 / 2 \mathrm{H}$, table 3). Expected values have been calculated under a ratio $1: 4 \mathrm{E}-\mathrm{E}: \mathrm{t}-\mathrm{E}$

\begin{tabular}{|c|c|c|c|c|c|}
\hline Plant & $\begin{array}{l}\text { Chromosome } \\
\text { arm }\end{array}$ & & $E-E$ & $t-E$ & $\chi^{2}$ \\
\hline $\mathrm{tE} 2$ & $1 \mathrm{RS}$ & $\begin{array}{l}\text { obs. } \\
\text { exp. }\end{array}$ & $\begin{array}{l}29 \\
19 \cdot 2\end{array}$ & $\begin{array}{l}67 \\
76 \cdot 8\end{array}$ & $6 \cdot 25^{*}$ \\
\hline & $3 R S$ & $\begin{array}{l}\text { obs. } \\
\text { exp. }\end{array}$ & $\begin{array}{l}42 \\
31 \cdot 0\end{array}$ & $\begin{array}{l}113 \\
124 \cdot 0\end{array}$ & $4 \cdot 88^{*}$ \\
\hline $\mathrm{tE} 3$ & $1 \mathrm{RS}$ & $\begin{array}{l}\text { obs. } \\
\text { exp. }\end{array}$ & $\begin{array}{l}40 \\
19 \cdot 2\end{array}$ & $\begin{array}{l}56 \\
76 \cdot 8\end{array}$ & $28 \cdot 17^{* *}$ \\
\hline
\end{tabular}

* Significant at the level of 5 per cent.

** Significant at the level of 1 per cent.

Dvốák and McGuire (1981) suggested that changes in chromosomes at the level of nucleotide sequence might be recognizable during the synaptic process. This kind of chromosome differentiation may lead to preferential pairing between more similar partners not only in allopolyploids, in which homologous and homoeologous chromosomes compete for pairing, but even in polyploids where homologues derived from a certain inbred line or cultivar (called "euhomologues") vie with homologues from a different origin within the same taxon ("heterohomologues") (Reinbergs et al., 1970). Thus identical pairing preferences found here could be ascribed to cytologically undetectable differences between heterohomologues from lines $\mathrm{t}$ and $\mathrm{E}$.

The homologous over identical pairing preferences detected in autotetraploid rye by Santos et al. (1983) and Orellana and Santos (1985) could be explained neither by differences in pairing activity nor by chromosome differentiation between homologues since under these assump- tions preferential pairing always will involve more similar partners, i.e., identical over homologous preferential pairing. Those authors pointed out that the existence of preferences mostly depends on the pairing affinities among chromosomes in a competitive situation. There is a subtle but essential difference between the terms "activity" (or ability) and "affinity"; generally, activity is referred to as a property of one specific chromosome for pairing with any other, whereas affinity is related to the capacity of two specific partners for pairing each other. Giraldez and Santos (1981) and Santos et al. (1983) suggested that differential pairing affinities may be due to the existence of genotypic or structural differences between chromosomes; in some cases these differences might lead to a higher affinity between less similar partners (homologous over identical preferential pairing). All the results obtained here are also explainable under this point of view.

Otherwise our results might be interpreted as a reflection of a closer proximity between identical partners after chromosome doubling; however, due to the time when the colchicine treatment was made, the cells in which polyploidy arose underwent many mitosis events before the resulting cells entered meiosis, making unlikely that high proximity between identical chromosomes was maintained during these processes.

Acknowledgements This work has been supported by a grant from the Comisión Asesora de Investigación Científica y Técnica of Spain.

\section{REFERENCES}

ABIRACHED-DARMENCY, M., CAUDERON, D. AND ZICKLER, Y. 1983. Synaptonemal complex and recombination nodules in rye (Secale cereale). Chromosoma, 88, 299306.

BENAVENTE, E. AND ORELlanA, J. 1985. Evidence for preferential pairing in telotrisomic plants of rye. Heredity, $55, " 181-186$.

BENAVENTE, E. AND ORELlaNA, J. 1986 Pairing competition between identical and homologous chromosomes in diploid and tetraploid cells of rye telotrisomic plants. Can. J. Genet. Cytol., 28, 568-573.

COMINGS, D. E. 1972. The structure and function of chromatin. Adv. Hum. Genet., 3, 237-431.

DOYLE, G. G. 1979. The allotetraploidization of maize. Part 1: The physical basis-differential pairing affinity. Theor. Appl. Genet., 54, 103-112.

DVORAK, J. AND McGUIRE, P. E. 1981. Nonstructura! chromosome differentiation among wheat cultivars, with special reference to differentiation of chromosomes in related species. Genetics, 97, 391-414. 
GILLIES, C. B. 1985 An electron microscopic study of synaptonemal complex formation at zygotene in rye. Chromosoma, 92, 165-175.

giraldez, R., Cermeño, M. C. AND ORellana, J. 1979. Comparison of C-banding pattern in the chromosomes of inbred lines and open pollinated varieties of rye. $Z$. Pflanzenzüchtg., 83, 40-48.

GIRALDEZ, R. AND SANTOS, J. L. 1981. Cytological evidence for preferences of identical over homologous but not-identical meiotic pairing. Chromosoma, 82, 447-451.

JACKSON, R. C. AND HAUBER, D. P. 1982. Autotriploid and autotetraploid cytogenetic analyses: correction coefficients for proposed binomial models. Am. J. Bot., 69, 644-646.

JOHN, B. AND HENDERSON, S. A. 1962. Asynapsis and polyploidy in Schistocerca paranensis. Chromosoma, 13, 111-147.

NARANJO, T. AND ORELLANA, J. 1984. Meiotic behaviour of chromosomes $1 \mathrm{R}, 2 \mathrm{R}$ and $5 \mathrm{R}$ in autotetraploid rye. Chromosoma, 89, 143-150.

ORELlANA, J, AND SANTOS, J. L. 1985. Pairing competition between identical and homologous chromosomes in autotetraploid rye. I. Submetacentric chromosomes. Genetics, 111, 933-944.

PARKER, J. S. 1987. Increased chiasma frequency as a result of chromosome rearrangement. Heredity, 58, 87-94.

PARKER, J. S., PALMER, R. W., WHITEHORN, M. A. F. AND EDGAR, L. A. 1982. Chiasma frequency effects of structural chromosome change. Chromosoma, 85, 673-686.
REINBERGS, E., KAO, K. N., HARVEY, B. L. AND SHEBESK1, L. H. 1970. Meiotic behavior and preferential pairing in autotetraploid barley. Crop Sci., 10, 569-571.

SANTOS, J. L., ORfllana, J. AND GiRAldeZ, R. 1983. Pairing competition between identical and homologous chromosomes in rye and grasshoppers. Genetics, 104, 677-684.

SChlfgel, R., MEI.Z, G. AND NESTROWICZ, R. 1987. A universal reference karyotype in rye, Secale cereale $\mathrm{L}$. Theor. Appl. Genet., 74, 820-826.

STACK, S. M. 1984. Heterochromatin, the synaptonemal complex and crossing over. J. Cell Sci, 71, 159-176.

SVED, J. A. 1966. Telomere attachment of chromosomes, some genetical and cytological consequences. Genetics, $53,747-756$.

SYBENGA, J. 1966. The zygomere as hypothetical unit of chromosome pairing. Genetica, 37, 186-198.

SyBenGA, J. 1975. Meiotic configurations. Monogr. Theor. Appl. Genet., vol. 1. Springer-Verlag, Berlin, Heidelberg, New York.

THOMAS, H. M. ANI PICKERING, P. A. 1979. Barley $\times$ rye crosses: the morphology and cytology of the hybrids and the amphiploids. Z. Pflanzenzüchtg., 82, 193-200. 